Artículo

\title{
Cartografía del aguacate en el sur del estado de México mediante tratamiento digital de imágenes sentinel-2
}

Dulce Karen Figueroa-Figueroa ${ }^{1}$

José Francisco Ramírez-Dávila ${ }^{\S}$

Xanat Antonio-Némiga ${ }^{2}$

Andrés González Huerta ${ }^{1}$

${ }^{1}$ Facultad de Ciencias Agrícolas-Universidad Autónoma del Estado de México. Carretera Toluca-Ixtlahuaca km 15.5, El Cerrillo Piedras Blancas, Toluca, Estado de México, México. CP. 50295. (dk_ figueroa@hotmail.com; agonzalezh@uaemex.mx). ${ }^{2}$ Facultad de Geografía-Universidad Autónoma del Estado de México. Cerro Coatepec s/n, Ciudad Universitaria, Toluca Estado de México, México. CP. 50110. (xanynemiga@ hotmail.com).

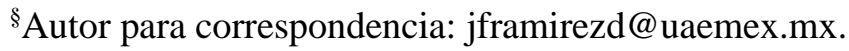

\section{Resumen}

El cultivo de aguacate (Persea americana Mill.) es uno de los más importantes en México, entre los estados con mayor producción se encuentra el Estado de México, que es el tercer estado productor a nivel nacional. Coatepec Harinas y Donato Guerra son dos de los municipios más representativos en lo respectivo a esta actividad; sin embargo, no existe un censo que especifique la superficie del cultivo, por lo que el objetivo de esta investigación fue probar métodos de índices de vegetación, algoritmos spectral angle mapper (SAM) y spectral information divergence (SID) y la combinación de estos en las imágenes del sensor Sentinel-2 para evaluar su desempeño en la identificación de áreas plantadas con el cultivo de aguacate. Los resultados se validaron con una matriz de confusión y la comparación de los datos de referencia de entrenamiento y validación. El algoritmo SID alcanzó una precisión de $97.5 \%$ para detectar aguacate, mientras que el tratamiento SAM obtuvo una precisión de $63.1 \%$. La combinación de SID con el índice Anthocyanin Reflectance Index 1 (ARI1), proporcionó un mejor resultado sobre la cartografía de validación regional con $85 \%$ de precisión. Otras combinaciones de índices y tratamientos dieron resultados inferiores al $50 \%$ de la precisión por lo que no se recomiendan. Esta metodología podría ser probada para la detección de otros cultivos de interés comercial, dado que Sentinel-2 muestra ser una alternativa viable para este tipo de estudios, teniendo una buena resolución espectral, además de ser de fácil acceso y manipulación.

Palabras clave: Persea americana Mill., índices de vegetación, SAM.

Recibido: enero de 2020

Aceptado: marzo de 2020 


\section{Introducción}

México es el principal productor de aguacate en el mundo, con una producción promedio anual de 1836700 t, en 235317 ha (SIAP, 2019), la entidad líder en producción nacional sigue siendo Michoacán. En segundo y tercer lugar respectivamente, se encuentran Jalisco y el Estado de México, este último contribuye con grandes superficies de plantaciones de aguacate, las cuales se distribuyen en diferentes municipios al sur del estado: Coatepec Harinas 2155 ha, Tenancingo 884 ha, Temascaltepec 1558 ha, Donato Guerra 1493 ha y Villa Guerrero 1321 ha (SIAP, 2019).

Actualmente, el aguacate Hass absorbió la producción, ya que del total de hectáreas que se dedican a la siembra de aguacate en el Estado de México, entre 90 y 95\% están destinadas a esta fruta (Sangerman et al., 2014). Este cultivo ha originado cambios profundos en la cultura agrícola de la región, ya que áreas anteriormente sembradas con maíz fueron ocupadas paulatinamente por huertos de aguacate; asimismo, ha traído una serie de consecuencias en los pueblos y comunidades de la región (Chávez, 2012).

La importancia del impacto económico que tiene el cultivo de aguacate en el Estado de México, así como el diagnóstico de la superficie que ocupa para una gestión eficiente y sostenible, hace del uso de los métodos de teledetección una opción viable gracias a su potencial en la identificación de cultivos agrícolas y forestales específicos, por lo que Sentinel-2 son particularmente atractivas, debido a su libre disponibilidad, con resolución espacial relativamente alta, así como bandas posicionadas estratégicamente, útiles para muchas aplicaciones que incluyen caracterización y cartografía de la vegetación (Chemura et al., 2018), así como llevar a cabo el seguimiento de una serie de cambios de uso de suelo y vegetación, por lo que el satélite es de interés para cultivos (ESA, 2017).

Los índices de vegetación son uno de los fundamentos de la teledetección, que reside en una respuesta diferente de las distintas superficies a las diferentes longitudes de onda del espectro electromagnético (Pérez, 2017). Cada tipo de vegetación responde de diferente forma (Chuvieco, 2007), se cumplen parámetros generales, dados por una baja reflectividad en el visible, alta en el infrarrojo cercano (NIR) y baja con presencia de algunos picos en el infrarrojo de onda corta (SWIR). Estos índices de vegetación consisten en la abstracción o síntesis de los valores de las bandas de la imagen a una sola banda mediante operaciones aritméticas, el resultado es ajustado normalmente a una escala de 0 a 1, pero puede variar (Chemura et al., 2016).

Debido a las características de Sentinel-2 y la importancia económica del cultivo de aguacate y su distribución, el objetivo de esta investigación fue probar métodos de índices de vegetación, algoritmos spectral angle mapper (SAM) y spectral information divergence (SID) y la combinación de estos en las imágenes de acceso libre del sensor Sentinel-2 para evaluar su desempeño en la identificación de áreas plantadas con el cultivo de aguacate. Se considera de gran valor contar con información detallada de la distribución y superficie de este cultivo para mostrar a los interesados información más cercana a la realidad y así plantear estrategias de gestión más eficaces. 


\section{Materiales y métodos}

Se aplicaron los métodos de índices de vegetación, algoritmos SAM y SID y la combinación de estos últimos con los índices de vegetación en las imágenes de acceso libre del sensor Sentinel-2 de mayo de 2017, re-muestreadas a una resolución espacial de $10 \mathrm{~m}$, libre de nubes y a un nivel de procesamiento $1 \mathrm{C}$; es decir, en valores de reflectancia en el tope de la atmósfera (TOA).

Para la identificación del cultivo de interés, se realizó con la metodología de tratamiento de la imagen, obteniendo un resultado individual del análisis espectral de la firma del aguacate con los algoritmos spectral angle mapper (SAM) y spectral information divergence (SID), el uso de índices de vegetación y la combinación de los algoritmos con los índices de vegetación.

\section{Área de estudio}

El estudio se realizó en dos municipios del sur del Estado de México (Figura 1). Coatepec Harinas forma parte del segundo sistema del Xinantécatl por lo que su altitud varia de 1900 a $3000 \mathrm{~m}$, su clima es templado subhúmedo, con temperatura promedio es de $16.1^{\circ} \mathrm{C}$ y precipitación promedio anual de $1242.53 \mathrm{~mm}$ por lo que predomina el bosque mesófilo de montaña.

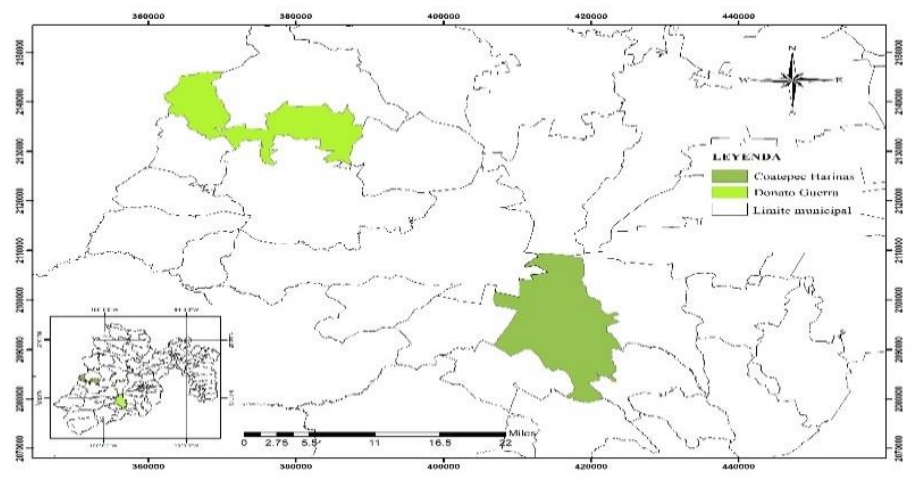

Figura 1. Área de estudio.

Donato Guerra se ubica en la región que pertenece al Eje Neovolcánico Transversal, por lo que presenta formas características de relieve accidentado, semiplano y plano, gran parte de las sierras son de origen volcánico con altitud entre 2200 y $3040 \mathrm{~m}$, su clima es templado subhúmedo con temperatura promedio de $22{ }^{\circ} \mathrm{C}$ y precipitación promedio de $1000 \mathrm{~mm}$ (PMDUCHEM, 2009; PMDUDGEM, 2009).

\section{Datos de campo}

Se aplicaron dos conjuntos de datos de referencia, uno para entrenamiento y otro para validación. Los datos de campo para el proceso de entrenamiento se obtuvieron el 18 de mayo de 2017 con un GPS Garmin ${ }^{\circledR}$ digital portátil, logrando precisiones entre tres y cinco metros, los cuales consistieron en 130 puntos de datos de campo georreferenciados para el cultivo de aguacate, distribuidos aleatoriamente en el área de estudio. 
Respecto al conjunto de datos para validación, se georreferenciaron 120 puntos en áreas de cada municipio totalmente alejadas de los primeros puntos de muestreo (entrenamiento), por lo que cabe mencionar que este conjunto de datos es totalmente independiente del primer conjunto de datos y no fue utilizado para entrenamiento de la imagen Sentinel-2. Además de que en la zona de estudio se cuenta con suelos leptosoles.

\section{Adquisición de imágenes y preprocesamiento}

Los datos de Sentinel-2 se descargaron del servidor de la agencia espacial europea (ESA) disponible en: https://scihub.copernicus.eu/dhus/: consultada el 06 de junio de 2017 con el nombre de escena: S2B_MSIL1C_20171128T170639_N0206_R069_T14QMG_20171128T221610,S2B_ MSIL1C_20171128T170639_N0206_R069_T14QLG_20171128T221610;S2B_MSIL1C_2017112 8T170639_N0206_R069_T14QMF_20171128T221610 correspondientes al 7 de mayo de 2017, fecha en la que se obtuvieron imágenes libres de nubes. Las imágenes fueron remuestreadas a una resolución espacial de $10 \mathrm{~m}$ con el software libre SNAP 5.0 desarrollado y distribuido por la Agencia Espacial Europea (ESA).

La imagen se calibró radiométricamente mediante el método de FLAT FIELD CALIBRATION, se marcó un área de interés ROI (Region of Interest) haciendo uso del techo plastificado de los invernaderos como referencia, debido a que emiten una respuesta alta y homogénea y se agregó la longitud promedio de onda de cada banda con el software ENVI 4.7.

\section{Procesamiento de la imagen Sentinel-2}

La imagen fue procesada mediante dos estrategias: el uso del clasificador spectral angle mapper (SAM) y spectral information divergence (SID) y el desarrollo de consultas lógicas en los índices de vegetación. La Figura 2, muestra el proceso del método combinado aplicado a la imagen Sentinel-2 MSI para obtener los sitios con cultivo de aguacate en la zona de estudio.

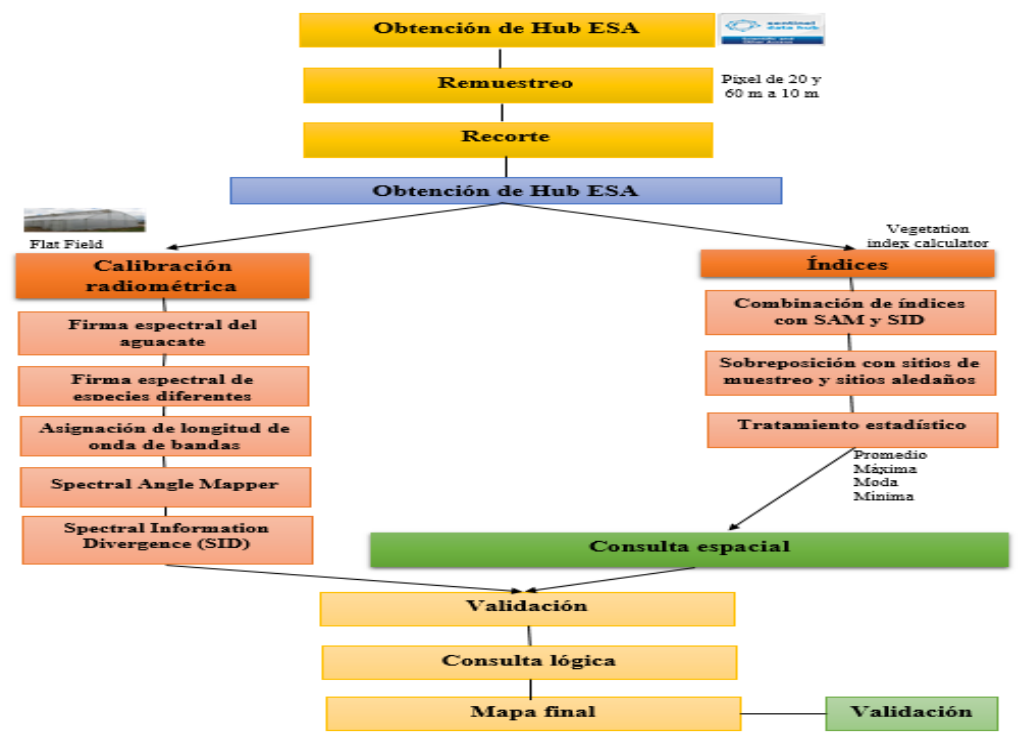

Figura 2. Proceso del método aplicado a la imagen Sentinel-2. 
Se obtuvo la curva espectral típica del cultivo de aguacate y de las librerías excluidas se excluyó la vegetación de laurel, cordillera de jaspe, madroño y dos variedades de roble con lo cual se obtuvo la firma espectral (Figura 3).

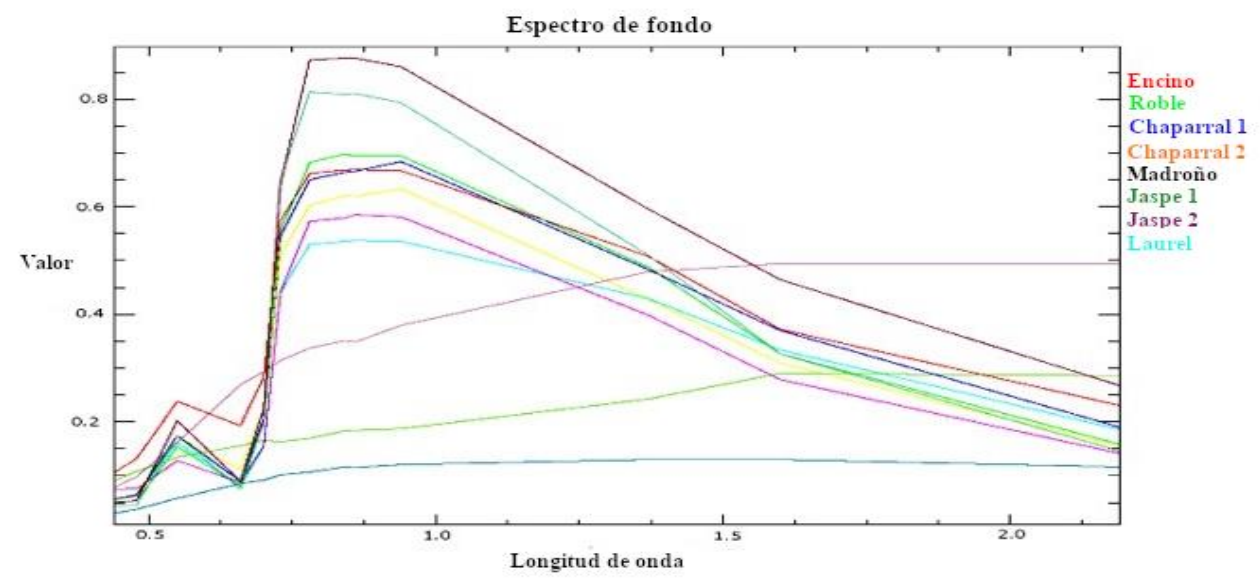

Figura 3. Librerías excluidas.

Estas firmas espectrales se guardaron como referencia y se emplearon para clasificar la imagen considerando el algoritmo SAM y SID. Se generaron reglas de confiabilidad con las firmas espectrales obtenidas del cultivo de aguacate de acuerdo con su clasificación y se llevaron a cabo pruebas para visualizar cual generaba un resultado más claro. Respecto al ángulo elegido para SAM y SID, el cual produce una imagen clasificada basada en el umbral de ángulo máximo de este, se probaron los ángulos máximos de 0.1, 0.075, 0.050 y 0.025.

Se realizó una clasificación de plantaciones encontradas en campo de acuerdo con la reportada por Rubí et al. (2013) árboles de 1 a 3 años (Figura 4), 3 a 6 años (Figura 5) y la tercera de más de 6 años en adelante (Figura 6) que demuestran mayor distancia en las bandas 8, 10 y 12. Se obtuvo la curva del comportamiento de cada clasificación de las plantaciones.

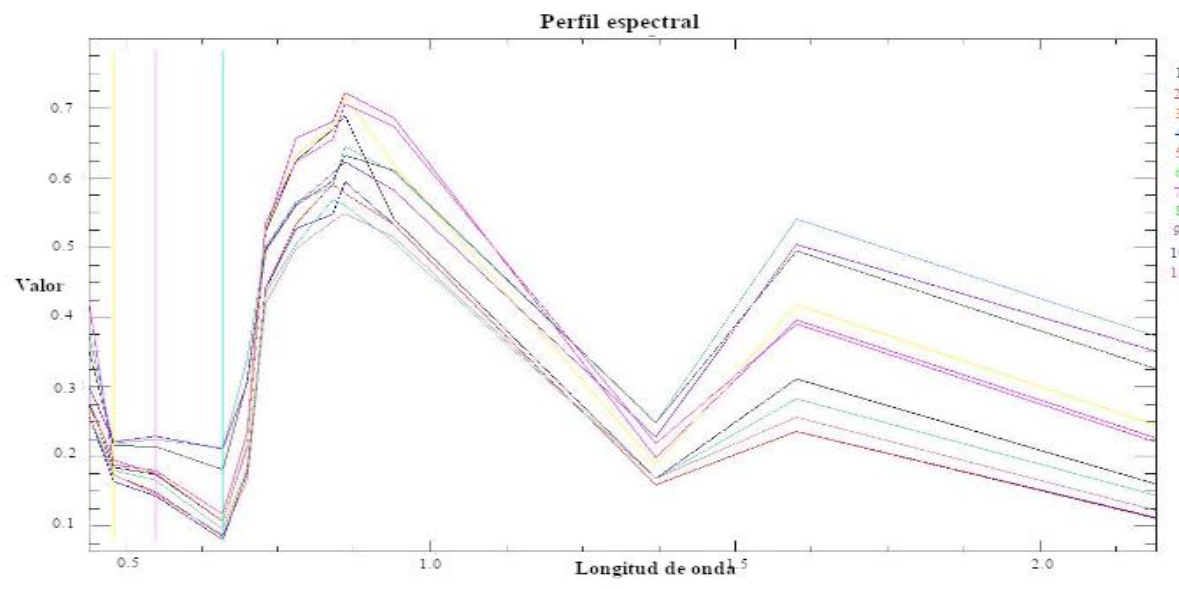

Figura 4. Árboles de 1 a 3 años. 


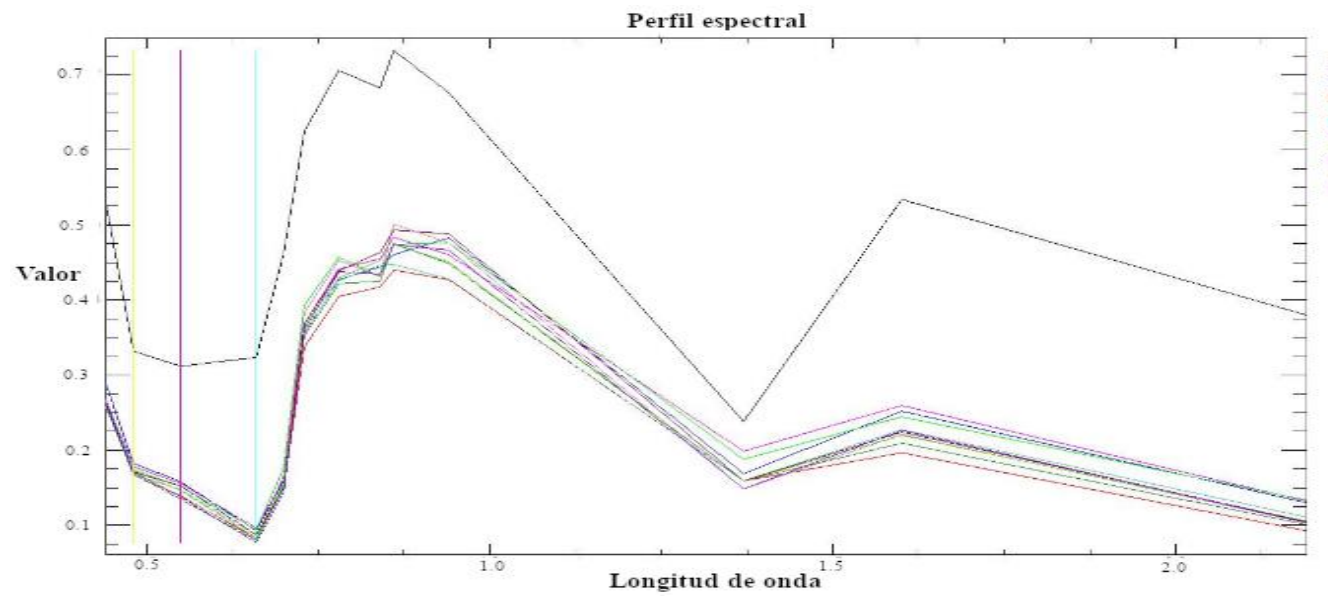

Figura 5. Árboles de 3 a 6 años.

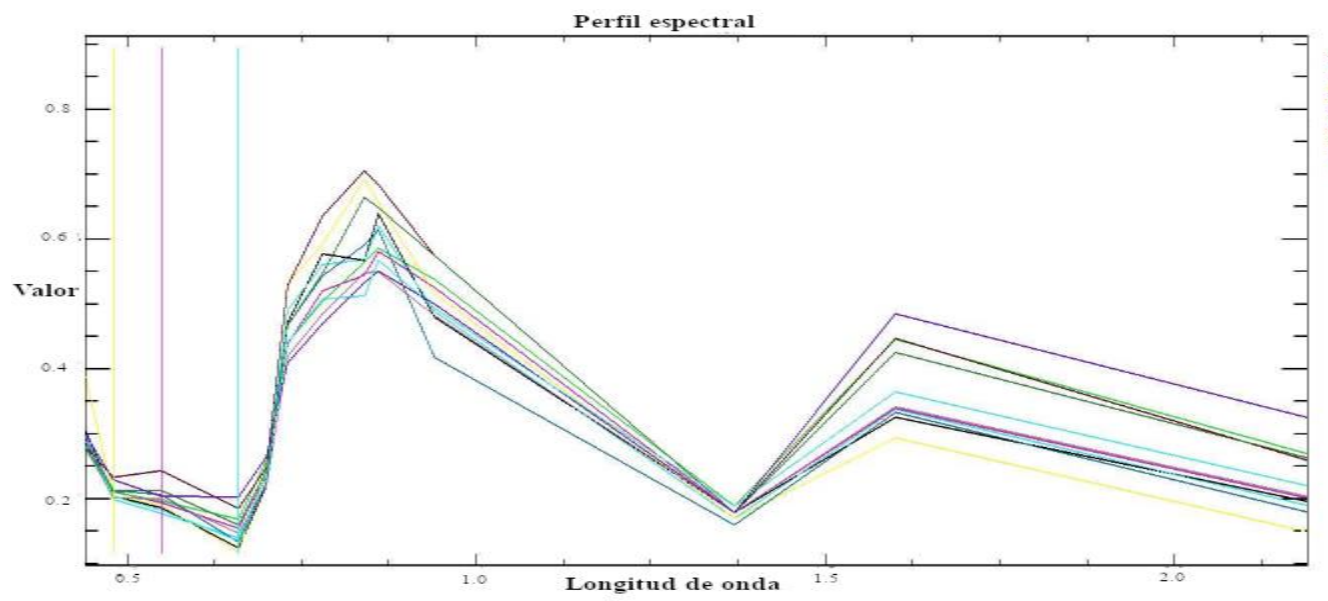

Figura 6. Árboles de 6 años en adelante.

Con la finalidad de aplicar cada uno de los algoritmos e índices de vegetación de acuerdo con el tipo de plantaciones presentes en el área de estudio de las cuales se eligieron solo diez árboles que presentaron la curva típica del cultivo de aguacate (Figura 7) de comportamiento de cada uno de los patrones espaciales correspondientes a plantaciones.

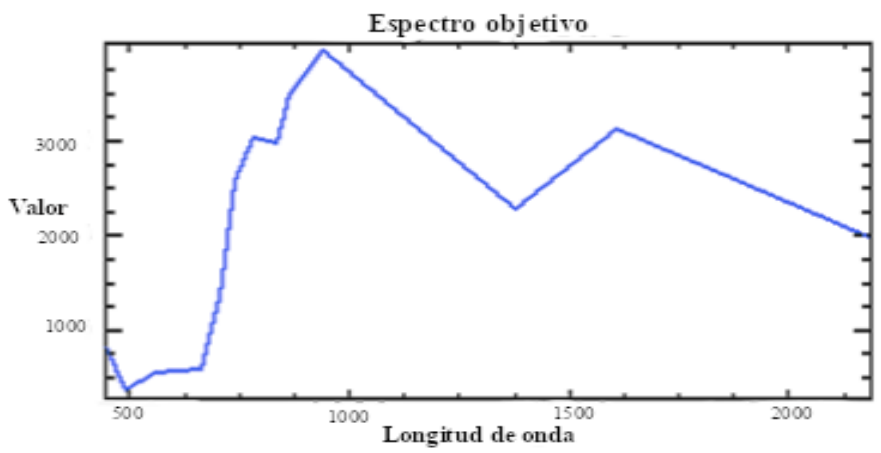

Figura 7. Curva típica del aguacate. 
Para la identificación de áreas con cultivo de aguacate se aplicó a los algoritmos SAM y SID los diferentes valores de ángulo máximo (radianes) elegidos con base en la prueba y error (Cuadro 1).

\section{Cuadro 1. Algoritmo y ángulo aplicado.}

\begin{tabular}{lll}
\hline Algoritmo & Lugar y tipo de plantación & Angulo máximo \\
\hline SAM & Coatepec plantaciones de 1 a 3 años & $0.1,0.075$, \\
SID & Coatepec plantaciones de 3 a 6 años & $0.05,0.025$ \\
& Coatepec plantaciones de más de 6 años & \\
SAM & Donato plantaciones de 1 a 3 años & $0.1,0.075$, \\
SID & Donato plantaciones de 3 a 6 años & $0.05,0.025$ \\
& Donato plantaciones de más de 6 años & \\
\hline
\end{tabular}

\section{Índices de vegetación}

Con el software Envi 4.7 se probaron 10 índices de vegetación disponibles de los que se obtuvieron cuatro índices de vegetación que proporcionaron mejor resultado: anthocyanin reflectance index 1 (ARI1), anthocyanin reflectance index 2 (ARI2), normalized difference vegetation index (NDVI) y simple ratio index (SR) (Cuadro 2).

Cuadro 2. Índices de vegetación calculados y relación de bandas 'B' empleadas.

\begin{tabular}{cccc}
\hline Índice & Abreviatura & Bandas Sentinel-2 & Autor \\
\hline Anthocyanin reflectance index 1 & ARI1 & ARI1 $=\frac{1}{\mathrm{~B} 3}-\frac{1}{\mathrm{~B} 5}$ & \\
Anthocyanin reflectance index 2 & ARI2 & ARI2 $=\frac{\mathrm{B} 8}{\mathrm{~B} 2}-\frac{\mathrm{B} 8}{\mathrm{~B} 3}$ & $\begin{array}{c}\text { Sentinel-Hub } \\
(2017)\end{array}$ \\
Normalized difference vegetation index & NDVI & NDVI $=\frac{\mathrm{B} 8-\mathrm{B} 4}{\mathrm{~B} 8+\mathrm{B} 4}$ & \\
Simple ratio index & & $\mathrm{SR}=\frac{\mathrm{B} 8}{\mathrm{~B} 4}$ & \\
\hline
\end{tabular}

En el software ArcGis 10.2.2, se analizaron los valores mínimo, medio y máximo de estos índices en sitios con y sin aguacate. Una vez entendido los valores característicos del aguacate, se construyeron consultas lógicas por medio del valor de cada pixel de la escena para extraer los valores de los índices, con los criterios que a continuación se despliegan (Cuadro 3 y 4).

Cuadro 3. Valor de la condicional aplicada a cada uno de los índices.

\begin{tabular}{lll}
\hline Índice & Lugar y tipo de plantación & Consulta condicional aplicada \\
\hline ARI1 & Coatepec de 1 a 3 años & Value $>0.2$ value $<1.45$ \\
& Coatepec de más de 6 años & Value $>=0.39$ \\
& Donato de 3 a 6 años & Value $>=0.3$ value $<=1.45$ \\
\hline
\end{tabular}




\begin{tabular}{lll}
\hline Índice & Lugar y tipo de plantación & Consulta condicional aplicada \\
\hline ARI2 & Coatepec de 1 a 3 años & Value $>=0.35$ value $<=0.82$ \\
& Donato de 3 a 6 años & Value $>=0.34$ value $<=0.95$ \\
& Donato de más de 6 años & Value $>0.07$ value $<0.65$ \\
\multirow{2}{*}{ NDVI } & Coatepec de 3 a 6 años & Value $>=0.5$ and value $<=0.73$ \\
& Donato de más de 6 años & Value $>0.4$ and value $<0.65$ \\
SR & Donato de más de 6 años & Value $>=2.45$ \\
\hline
\end{tabular}

Se asignó el valor 1 para los pixeles que cumplen con la condición y 0 para los pixeles $\sin$ valor. Value $=$ valor del pixel.

Cuadro 4. Valor de la condicional aplicada a las combinaciones de índices y algoritmos.

\begin{tabular}{lll}
\hline Combinaciones & Lugar y tipo de plantación & Consulta condicional aplicada \\
\hline SAM + ARI1 & Coatepec de 3 a 6 años & Value $>=0.025$ and value $<=0.65-1.35$ \\
SAM + NDVI & Coatepec de 3 a 6 años & Value $>=0.1$ and value $<=0.5-0.73$ \\
SID + ARI1 & Coatepec de 1 a 3 años & Value $>=0.05$ and value $<=0.2-0.145$ \\
SID + ARI1 & Donato de 3 a 6 años & Value $>=0.1$ and value $<=03-0.145$ \\
SID + ARI 2 & Donato de 3 a 6 años & Value $>=0.1$ and value $<=0.34-0.95$ \\
SID + NDVI & Coatepec de 3 a 6 años & Value $>=0.05$ and value $<=0.5-0.73$ \\
SID + SR & Coatepec de 3 a 6 años & Value $>=0.075$ and value $<=0.5-0.73$ \\
& Coatepec de 3 a 6 años & Value $>=0.1$ and value $<=0.5-0.73$ \\
& Donato de 3 a 6 años & Value $>=0.1$ and value $<=2.45$ \\
Algoritmos & Donato de 3 a 6 años & Value $>=0.075$ and value $<=2.45$ \\
SAM & Coatepec de 3 a 6 años & Ángulo \\
SID & Donato de más de 6 años & Value $=0.1,0.075,0.05,0.0025$ \\
\hline Se & Value $=0.1,0.075,0.05,0.0025$
\end{tabular}

Se asignó el valor 1 para los pixeles que cumplen con la condición y 0 para los pixeles sin valor.

Para la cartografía de los cultivos, no necesariamente se consideró el mismo rango de las condicionales para todas las escenas, dado que los valores del índice pueden variar de escena a escena, por lo que se encontró el umbral de valores de índice que corresponden con el cultivo de interés. Las regiones extraídas, tanto del clasificador como de los índices se relacionaron mediante álgebra de mapas, con la herramienta COMBINATORIAL AND. Esto permitió consultar aquellos sitios donde ambos (el clasificador SAM y SID y la condicional de los índices) tienen un valor verdadero.

\section{Validación de mapas}

La precisión obtenida por estos tratamientos digitales en la imagen se validó mediante la sobre posición de esta cartografía contra puntos de validación obtenidos en campo, este conjunto de datos de validación permitió realizar una matriz de confusión (Cuadro 5) con la que se obtuvo el valor en porcentaje de la precisión de los tratamientos y el error de comisión, en el que se observa que tanto el algoritmo SID como sus combinaciones, presentan el mayor porcentaje de precisión para la obtención de zonas con plantaciones de aguacate en el área de estudio. 
Cuadro 5. Matriz de confusión de combinaciones de SAM, SID e índices de vegetación.

\begin{tabular}{cccccccc}
\hline \multicolumn{2}{c}{ Número de pixeles } & & & \multicolumn{3}{c}{ Porcentaje } \\
\cline { 1 - 2 } \cline { 6 - 7 } Tratamiento & Acierto & Error & & Total & Acierto & Error & Error de comisión \\
\hline SAM & 76 & 44 & & 120 & 63.1 & 36.6 & 6.7 \\
SID & 117 & 3 & & 120 & 97.5 & 2.5 & 13.2 \\
ARI1 & 103 & 17 & & 120 & 85.8 & 14.1 & 11.6 \\
ARI2 & 80 & 40 & & 120 & 66.6 & 33.3 & 18.7 \\
NDVI & 108 & 12 & & 120 & 90 & 10 & 31.7 \\
Simple Ratio & 95 & 25 & & 120 & 79.1 & 20.8 & 26.8 \\
SAM + ARI1 & 63 & 57 & & 120 & 52.5 & 47.5 & 2.44 \\
SAM + NDVI & 74 & 46 & & 120 & 61.6 & 38.3 & 4.2 \\
SID + ARI1 & 102 & 18 & & 120 & 85 & 15 & 9.7 \\
SID + ARI2 & 80 & 40 & & 120 & 66.6 & 33.3 & 41.1 \\
SID + NDVI & 108 & 12 & & 120 & 90 & 10 & 14.2 \\
SID + SR & 95 & 25 & & 120 & 79.1 & 20.8 & 23.6 \\
\hline
\end{tabular}

\section{Resultados y discusión}

Los resultados de la validación indican que la combinación con SID alcanzó una precisión de 97.5\% para detectar aguacate, mientras que el tratamiento SAM, obtuvo una precisión de 63.1\%, siendo SID un algoritmo más efectivo que SAM, tanto de forma individual como combinada con los índices de vegetación, por lo que coincidimos con lo mencionado por Du et al. (2004) que SID es un método de clasificación espectral que utiliza una medida de divergencia para hacer coincidir la respuesta de los píxeles de la imagen con los espectros de referencia.

Así como con lo observado por Du et al. (2004), quien indica que el algoritmo SID puede caracterizar la similitud espectral y la variabilidad de manera más eficaz que otros algoritmos de clasificación, ya que ve cada espectro de píxeles como una variable aleatoria, y después mide la discrepancia de los comportamientos probabilísticos que existe entre dos espectros. Respecto a los índices de vegetación probados, los mayores porcentajes de acierto son ARI1 con una precisión de $85.8 \%$ y NDVI $90 \%$.

Dentro de las combinaciones de índices de vegetación con los algoritmos y el resultado de la matriz de confusión (Cuadro 5), los métodos que alcanzaron mayor precisión son: SID con los índices ARI1, ARI2, NDVI y SR con porcentaje de $85 \%, 66.6 \%, 90 \%$ y $79.1 \%$ respectivamente, a pesar de que la suma del algoritmo con el índice NDVI obtuvo un mayor porcentaje de precisión, en este caso no se recomienda debido a que tiende a sobreestimar la superficie establecida de plantaciones de aguacate, la combinación de SID más ARI2 muestra un resultado demasiado conservador subestimando la superficie de plantaciones de aguacate establecida actualmente (Figura 8 a 11). 


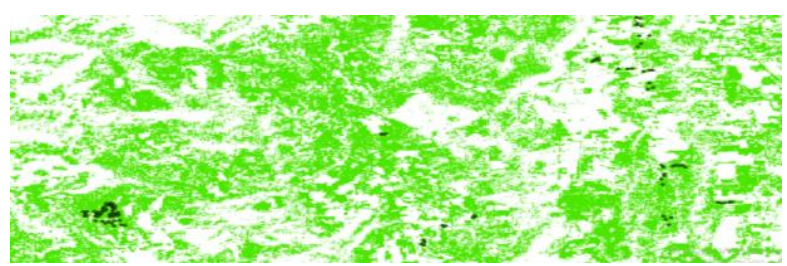

Figura 8. Combinación del índice SID + ARI1 plantaciones de 1 a 3 años y puntos de validación.

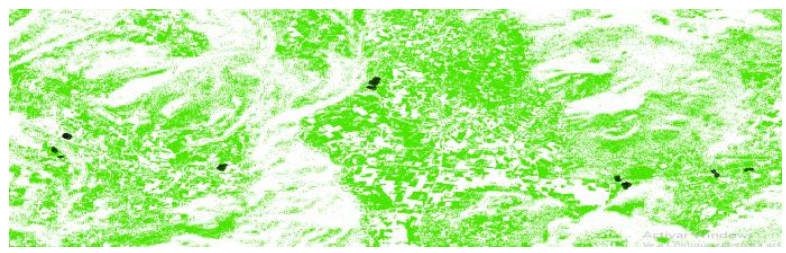

Figura 10. Combinación del índice SID + ARI2 plantaciones de 3 a 6 años y puntos de validación.

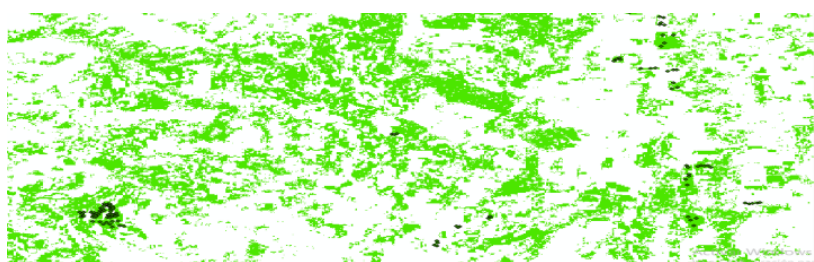

Figura 9. Combinación del índice SID + NDVI plantaciones de 3 a 6 años y puntos de validación.

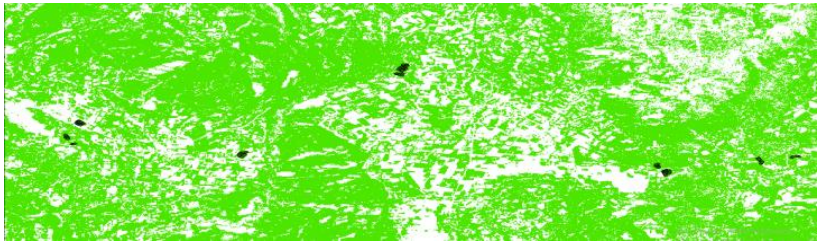

Figura 11. Combinación del índice SID + SR plantaciones de 3 a 6 años y puntos de validación.

Otras combinaciones de índices y métodos dieron resultados inferiores a $60 \%$ de la precisión, por lo que no se recomiendan. En lo que refiere a las combinaciones de los parámetros del ángulo espectral con los índices de vegetación se obtuvo una mejor respuesta con el ángulo máximo (radianes) $0.025,0.05$ y 0.075, resultando una imagen con mayor coherencia y mejor calidad, como lo menciona Robson et al. (2016) que la extracción adicional de información espectral específica muestra fuertes correlaciones entre los índices de vegetación derivados y una serie de parámetros medidos.

Los resultados obtenidos de los métodos combinados aplicados a la imagen Sentinel-2 muestran confiabilidad al exponer sitios con alta probabilidad de estar sembrados con cultivo de aguacate, lo que se corrobora con lo observado por, que indica que los resultados de la clasificación confirman el alto potencial de Sentinel-2 para obtener tipos de cultivos específicos y mapas de especies arbóreas.

En cuanto a la información espectral, la curva roja y ondas infrarrojas de onda corta (SWIR) para la cartografía de la vegetación, así como la banda azul fueron importantes para obtener la presencia del cultivo de aguacate en el área de estudio. El resultado de la presencia de superficie plantada de cultivo de aguacate en los municipios de Coatepec Harinas y Donato Guerra con $70 \%$ de precisión (Figuras 11 y 12) es de 5658.62 ha y 5901.57 ha, con un total de 11560.19 ha.

Dato que difiere de los reportados por SIACON (2017), quien reporta una superficie para estos dos municipios de 5501.36 ha, así como el SIAP (2017), que reporta 3188.36 ha para los dos municipios, para 2018 el SIAP reporta una superficie plantada de aguacate en Coatepec Harinas de 2155 ha y para Donato Guerra 1493 ha. La diferencia en los datos reportados de la superficie plantada del cultivo de aguacate en el área de estudio respecto a los obtenidos se atribuye a que las estadísticas oficiales tienen tres y un año de diferencia respecto a la información obtenida. 
Con los tratamientos aplicados a la imagen Sentinel-2, además de que existen reportes de que en los últimos años se han incrementado las plantaciones de aguacate en el área de estudio. Es importante mencionar que estas nuevas plantaciones se están estableciendo en zonas anteriormente cubiertas de bosque, lo que provoca la deforestación de estas, convirtiéndose en el origen de problemas ambientales de consideración a corto, mediano y largo plazo, tal como sucede en el estado de Michoacán, donde expertos señalan al cultivo de aguacate como el responsable de un ecocidio de magnitudes incalculables (Montiel et al., 2008; Toledo et al., 2009).

Las Figuras 12 y 13 muestran la cartografía del aguacate en Coatepec Harinas y Donato Guerra, Estado de México, obtenida mediante la combinación del SID y el índice ARI1, sobre el resultado obtenido de la combinación del algoritmo y el índice se sobrepusieron los puntos de validación obtenidos en campo. Los cuales se encuentran en áreas identificadas con presencia del cultivo de aguacate en ambos municipios, además de que se visualiza un patrón de comportamiento con establecimiento de plantaciones de cultivo de aguacate.

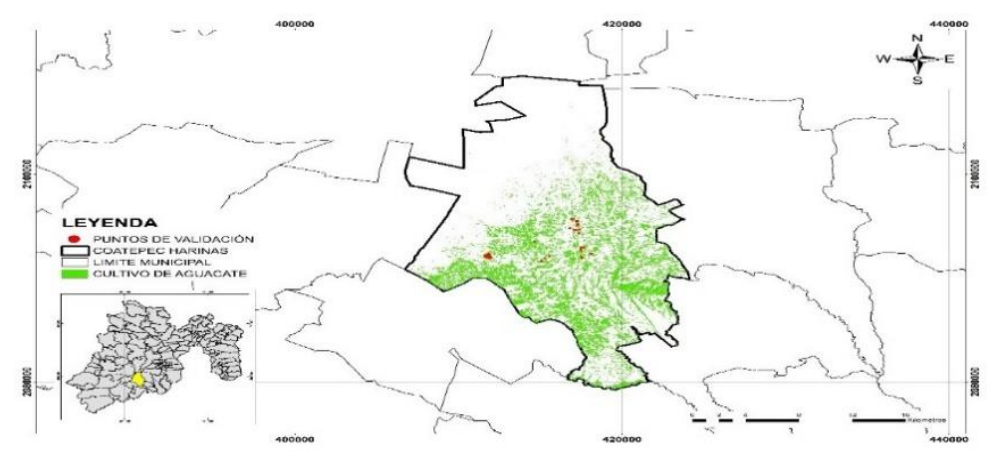

Figura 12. Cartografía del aguacate en Coatepec Harinas, Estado de México, obtenida mediante la combinación del SID y el índice ARI1.

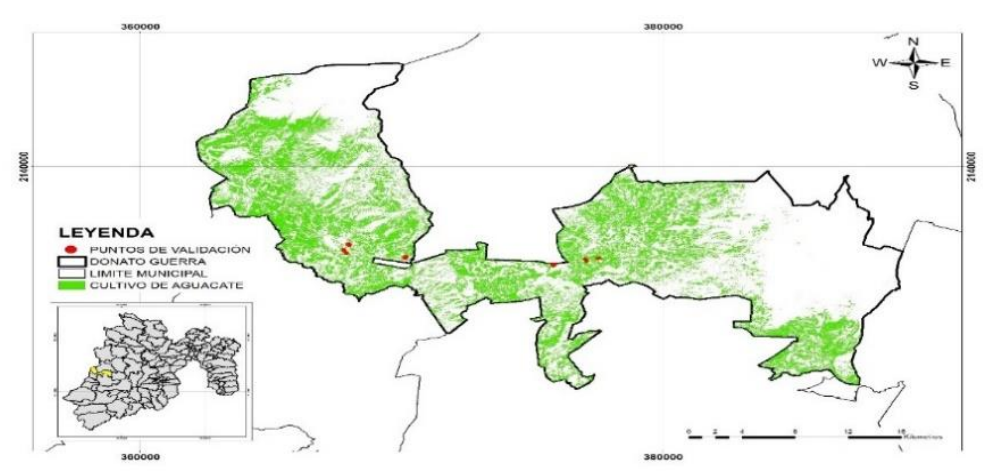

Figura 13. Cartografía del aguacate en Donato Guerra, Estado de México, obtenida mediante la combinación del SID y el índice ARI1.

En áreas con pendiente pronunciada y zonas donde anteriormente existía otro tipo de vegetación lo cual coincide con lo observado por Chávez et al. (2012) que la complejidad del cultivo del aguacate por la gran extensión que ocupa, su ciclo de producción, el uso de agroquímicos y el efecto sobre el entorno ambiental, hacen que este sistema-producto influya drásticamente en el cambio de uso del suelo y en el deterioro del medio ambiente en el que prospera. 
En la Figura 13 se observa que el resultado obtenido con estos métodos muestra un incremento de superficie plantada con el cultivo de aguacate hacia el noroeste del municipio de Donato Guerra lo cual puede estar influenciado por la colindancia con el estado de Michoacán. La vegetación que proporcionó resultados más cercanos a lo que sucede en campo fue la de 1 a 3 años y de 3 a 6 años con el ángulo 0.0025 y 0.0075 con la combinación SID más ARI1.

El índice de vegetación con mejor porcentaje de precisión tanto de manera individual como combinado con SID fue NDVI que obtuvo el $90 \%$ de precisión; sin embargo, cabe destacar que el resultado sobre la cartografía de validación a nivel regional se visualiza una sobreestimación de la presencia del cultivo de aguacate. Por lo que se atribuye al error de comisión, siendo la combinación de SID más ARI1 con $70 \%$ de precisión el que subestima de manera mínima la presencia del cultivo de aguacate. Se aprecia una mayor precisión debido a la presencia de una cantidad de pixeles más representativos en comparación a las otras combinaciones, esto se atribuye a la edad de las plantaciones que de acuerdo al índice y tipo de vegetación para determinarlo, los árboles de 1 a 3 años y de 3 a 6 años fueron los que mostraron un comportamiento uniforme tanto en la firma espectral como en el resultado obtenido.

El cambio en antiocianina, que es un pigmento que da los colores rojo, azul o púrpura a las plantas, en las hojas indica cambios en el follaje, crecimiento de brotes o muerte. Sin embargo, el debilitamiento de la vegetación contiene mayores concentraciones de antocianinas, por lo que este índice es una medida de la vegetación estresada. Afirmación con la cual se difiere, debido a que su comportamiento en plantaciones con edades de 1 a 3 años y de 3 a 6 años mostró buen desempeño.

Lo que se atribuye que los aumentos en ARI1 indican cambios en el follaje a través de un nuevo crecimiento, razón por la que este índice fue acertado. De acuerdo con el comportamiento del índice NDVI y su combinación con SID, en donde se obtuvo error de comisión y la vegetación y patrones no cumplieron totalmente con los requerimientos de este índice, se atribuye al observado por Gill et al. (2009) quien comenta que NDVI tiene problemas en la estimación de la cubierta vegetal.

Por ejemplo, este índice comienza a saturar cuando la cubierta vegetal es superior al 50\%, y no es sensible al verde cuando la cubierta vegetal es baja. Por este motivo puede sobre o subestimar la cubierta vegetal, dando información errónea en algunas zonas (Zhongming et al., 2009). Probablemente una de las explicaciones a la falta de precisión y errores en los índices y combinaciones aplicadas a esta investigación se deba a lo encontrado en varios estudios (Castro et al., 2004; Sánchez y Castro, 2006; Kalacska et al., 2007), al disponer de un tamaño de píxel de 10 $\mathrm{m}$, si la superficie ocupada por las copas del árbol es inferior a esta, pueden darse problemas de identificación de especies, por lo que una de las causas de no encontrar resultados satisfactorios. Podría ser la contaminación del pixel por especies trepadoras, epifitas o no estructurales dando lugar a confusión en la firma espectral característica de los árboles, como se ha demostrado en estudios llevados a cabo en bosques tropicales.

Autores como Abdulridha et al. (2019) en su investigación de detección de enfermedades en aguacate con teledetección menciona que la industria del aguacate se beneficiaría de un método automatizado y remoto detección para ayudar a los productores a tomar decisiones de gestión oportunas. Por lo que es importante reconocer que la tecnología de los sensores remotos que, dado su enfoque espacial exhaustivo y la temporalidad de las visitas a un mismo píxel, plantea una solución eficiente y de bajo costo (Zhang et al., 2012). 
Por lo que, la calidad de los resultados y la disponibilidad de la información hace a Sentinel-2 una alternativa viable y confiable para la identificación del cultivo de aguacate. Lo cual se puede respaldar adoptando medidas propuestas en varios estudios (South et al., 2004; Peña et al., 2008; Peña et al., 2011) en los que se menciona como la información sobre el uso de la tierra agrícola se actualiza de manera rutinaria en muchas regiones de cultivo en los EE. UU. y Europa; a través, de las comunicaciones de los agricultores o las visitas de inspectores administrativos a los campos seleccionados.

\section{Conclusiones}

Los métodos de índices de vegetación, algoritmos SAM y SID y la combinación de estos en las imágenes de acceso libre del sensor Sentinel-2, muestran alto potencial para la identificación de áreas plantadas con el cultivo de aguacate. La imagen Sentinel-2 procesada con el algoritmo SID tiene un gran potencial para estudios de identificación de vegetación, mostrando una alta resolución y precisión. El índice de vegetación ARI1 proporcionó mayor confiabilidad al determinar el patrón que sigue el cultivo de aguacate.

\section{Literatura citada}

Abdulridha, J.; Ehsani, E.; Abd-Erahman, A. and Ampatzidis, Y. 2019. A remote sensing technique for detecting laurel wilt disease in avocado in presence of other biotic and abiotic stresses. Computers and Electronics in Agriculture. 156:549-557.

Castro, K. L.; Sánchez, G. A. y Caelli, T. 2004. Discrimination of lianas and trees with leaf-level hyperspectral data. Remote Sensing of Environment. 90(3):353-372. Doi.org/10.1016/j.rse.2004.01.013.

Chavéz, L. G.; Tapia, V. L. M.; Bravo, E. M.; Sáenz, R. T.; Munoz, F. H. J. y Vidales, F. I. 2012. Impacto del cambio de uso de suelo forestal a huertos de aguacate. INIFAP. 13. ISBN: 978607-425-825-7. https://www.researchgate.net/publication/265125083_Impacto_del_ cambio_de_uso_del_suelo_forestal_a_huertos_de_aguacate_impact_of_forest_land_use_ change_to_avocado_orchards.

Chemura, A.; Mutanga, O. and Dube, T. 2016. Separability of coffee leaf rust infection levels with machine learning methods at Sentinel-2 MSI spectral resolutions. Precision Agric. 18(5):859-881. Doi:10.1007/s11119-016-9495-0.

Chemura, A.; Mutanga, O.; Odindi, J. and Kutywayo, D. 2018. Mapping spatial variability of foliar nitrogen in coffee (Coffea arabica L.) plantations with multispectral Sentinel-2 MSI data. ISPRS Journal of Photogrammetry and Remote Sensing. 138:1-11. Doi.org/10.1016/j.isprsjprs.2018.02.004.

Chuvieco, E. 2007. Teledetección ambiental. La observación de la tierra desde el espacio. Editorial Ariel, SA., Barcelona. 67-70 pp.

Du, Y.; Chang, C.; Ren, H.; Chang, C. and Jensen, J. 2004. New hyperspectral discrimination measure for spectral characterization. Optical Engineering. 43(8):1777-1786. Doi:10.1117/1.1766301.

ESA. 2017. European Space Agency. El programa Copérnico. http://www.esa.int/es1/ESA_in_your_ country/Spain/El_programa_Copertnico. 
Gill, T. K.; Phinn, S. R.; Armston, J. D. y Pailthorpe, B. A. 2009. Estimating tree-cover change in Australia: challenges of using the MODIS vegetation index product. International Journal of Remote Sensing. 30(6):1547-1565.

Kalacska, M.; Sánchez, A. G. A.; Rivard, B.; Caelli, T.; White, H. P. y Calvo, A. J. C. 2007. Ecological fingerprinting of ecosystem succession: estimating secondary tropical dry forest structure and diversity using imaging spectroscopy. Remote Sensing of Environment, 108(1):82-96. Doi.org/10.1016/j.rse.2006.11.007.

Montiel, A. L.; Krishnamurthy, A.; Vázquez, A. M. y Uribe, G. P. 2008. Opciones agroforestales para productores de aguacate. Terra latinoamericana. 26(1):234-244. http://www.scielo.org.mx/scielo.php?script=sci_arttext\&pid=S0187_57792008000100011 $\& \operatorname{lng}=\mathrm{es} \& \ln \mathrm{ln}=\mathrm{es}$.

Peña, B. J. M.; López, G. F.; García, T. L.; Jurado, E. M.; Sánchez, O. M. and García, F. A. 2008. Discriminating cropping systems and agro-enviromental measures by remote sensing. Agronomy for Sustainable Development. 28(2):355-362. doi.org/10.1051/agro:2007049.

Peña, B. J. M.; Ngugi, M. K.; Plant, R. E. and Six, J. 2011. Object-based crop identification using multiple vegetation indices, textural features and crop phenology. Remote Sensing of Environment. 115:1301-1316. Doi: 10.1016/j.rse.2011.01.009.

Pérez de la Cruz, M.; Hernández-May, M. A.; De la Cruz-Pérez, A. y Sánchez-Saul, S. 2017. Scolytinae y Platypodinae (Coleoptera: Curculionidae) de dos áreas de conservación en Tabasco, México. Revista de Biología Tropical. 64(1):335-342.

PMDUCH. 2009. Plan municipal de desarrollo urbano de Coatepec de Harinas, Estado de México. Datos estadísticos proporcionados por el DDR 078 Coatepec de Harinas. http://seduv.edomexico.gob.mx/planes_municipales/Coatepec de harinas.

PMDUDG. 2009. Plan municipal de desarrollo urbano de Donato Guerra, Estado de México. Datos estadísticos proporcionados por el DDR 078 Donato Guerra. http://seduv.edomexico.gob.mx/planes_municipales/Donato guerra.

Robson, A. J.; Petty, J.; Jayce, D. C.; Marques, J. R. and Hofman, P. J. 2016. High resolution remote sensing, GIS and Google Earth for avocado fruit quality mapping and tree number auditing. In: XXIX International Horticultural Congress on Horticulture: Sustaining Lives, Livelihoods and Landscapes (IHC2014): 1130:589-596.

Rubí, A. M.; Franco, M. A. L.; Rebollar, R. S.; Bobadilla, S. E. E.; Martínez, D. I. y Siles, H. Y. 2013. Situación actual del cultivo del aguacate (Persea americana Mill.) en el Estado de México. Tropical and Subtropical Agroecosystems. 16(1):93-101. http://www.redalyc.org/articulo.oa?id=93927469014.

Sangerman, J. D. M.; Larqué, S. B. S.; Omaña, S. J. M.; Shwenstesius, R. R. y Navarro, B. A. 2014. Tipología del productor de aguacate en el Estado de México. Rev. Mex. Cienc. Agríc. 5(6):1081-1095. http://www.redalyc.org/articulo.oa?id=263131532014.

Sánchez, A. G. A. and Castro, E. K. 2006. Canopy observations on the hyperspectral properties of a community of tropical dry forest lianas and their host trees. International Journal of Remote Sensing. 27(10):2101-2109. Doi.org/10.1080/01431160500444749.

SIAP. 2017. Servicio de Información Agroalimentaria y Pesquera. http://www.siap.gob.mx/.

SIAP. 2018. Servicio de Información Agroalimentaria y Pesquera. http://www.siap.gob.mx/.

SIAP. 2019. Servicio de Información Agroalimentaria y Pesquera. https://www.gob.mx/siap/ documentos/boletin-mensual-de-avances-de-la-produccion-de-aguacate-103935.

SIACON. 2017. Sistema de Información Agroalimentaria de Consulta. https:/www.gob.mx/ siap/prensa/sistema-de-informacion-agroalimentaria-de-consulta-siacon. 
South, S.; Qi, J. and Lusch, D. P. 2004. Optimal classification methods for mapping agricultural tillage practices. Remote Sensing of Environment. 91(1):90-97. Doi.org/10.1016/j.rse.2004.03.001.

Toledo, R.; Alcántar, J. J.; Anguiano, J. y Chávez, G. 2009. Expansión del cultivo del aguacate y deforestación en Michoacán. Boletín El Aguacatero. No. 58. https://www.sdpnoticias.com /negocios/deforestacion-contribuye-expansion-aguacate-cultivo.html.

Zhang, X.; Friedl, M. A.; Tan, B.; Goldberg, M. D. and Yu, Y. 2012. Long-term detection of global vegetation phenology from satellite instruments. 297-320 pp. In: X. Zhang (ed.). Chapter 16. Phenology and climate change. ISBN 978-953-51-0336-3. In tech open access publisher. Doi:10.5772/39197.

Zhongming, W.; Brian, G. L.; Jiao, F.; Wanning, L. and Haijing, S. 2009. Stratified vegetation cover index: A new way to assess vegetation impact on soil erosion. Catena. 83(1):87-93. Doi.org/10.1016/j.catena.2010.07.006. 